\title{
IDENTIFICAÇÃO DA DISFUNÇÃO TEMPOROMANDIBULAR (DTM) EM USUÁRIOS DE DISPOSITIVO DE PROTEÇÃO AUDITIVA INDIVIDUAL (DPAI)
}

\author{
Identification of the temporomandibular (tmd) dysfunction \\ in users of individual auditory protective devices (IAPD)
}

\author{
Hilda Santos de Souza Mendes Aquino ${ }^{(1)}$,Silvia Damasceno Benevides ${ }^{(2)}$, \\ Tatiana de Paula Santana da Silva ${ }^{(3)}$
}

\section{RESUMO}

Objetivo: descrever e classificar a presença de disfunção temporomandibular em trabalhadores usuários de equipamentos de proteção auditiva individual. Método: tratou-se de um estudo transversal descritivo, com caráter observacional, desenvolvido em uma indústria de tintas. A população foi composta por 46 funcionários de ambos os sexos (com 94,44\% do gênero masculino) com idade entre 20 a 50 anos (idade media de 36,24 anos), expostos a ruído intenso (90 dBNA) que faziam uso de equipamento de proteção individual auditiva tipo concha. Na coleta de dados foram realizados os seguintes procedimentos: triagem fonoaudiológica das funções orais, avaliação clínica otorrinolaringológica e diagnóstico de disfunção temporomandibular ao exame clínico. Os dados foram analisados pela estatística descritiva. Resultados: a queixa predominante foi dor na região que envolve a orelha e articulação temporomadibular. Observou-se que 87\% apresentaram diagnóstico positivo de DTM, em que $83 \%$ tinham dor miofacial, $11 \%$ dor miofacial com limitação de abertura oral e $6 \%$ apresentaram diagnóstico de dor miofacial associada a deslocamento de disco com redução de abertura. Conclusões: a maioria dos trabalhadores apresentou disfunção de ordem muscular. Ressalta-se a importância de considerar a possibilidade da influência deste equipamento sobre o aparecimento ou intensificação do transtorno mencionado.

DESCRITORES: Transtornos da Articulação Temporomandibular; Dor Facial; Saúde do Trabalhador; Proteção Auditiva Individual

\section{INTRODUÇÃO}

De acordo com a NR-6 quando esgotados todos os meios conhecidos para a eliminação do risco de acidentes ocupacionais e este persistir, mesmo que reduzido, é necessária a utilização, pelo trabalhador, de equipamentos de proteção auditiva individual

(1) Fonoaudióloga pela Universidade Católica de Pernambuco; Especialista em Audiologia pela Universidade Federal Pernambuco.

(2) Fonoaudióloga; Docente do curso de Graduação em Fonoaudiologia da Universidade Federal da Bahia; Mestre em Fisiologia pela Universidade Federal de Pernambuco.

(3) Fonoaudióloga; Mestranda em Hebiatria pela Universidade de Pernambuco.

Conflito de interesses: inexistente
(EPAI) que consistem em todos os dispositivos de uso individual que são destinados a proteger a integridade física do trabalhador, podendo ser utilizados na forma de vestimentas e equipamentos confeccionados nos mais diversos tipos de materiais ${ }^{1}$.

Os protetores auditivos são instrumentos utilizados nas indústrias. Estes são classificados basicamente em dois modelos: os circum-auriculares, também chamados extra-auriculares, supra-aurais ou "de concha" e os de inserção, intra-auriculares ou simplesmente conhecidos como "plugs" 2 .

Pesquisas na área da saúde auditiva do trabaIhador ${ }^{3,4}$ demonstram uma crescente preocupação com o conforto do trabalhador com relação ao uso dos EPI's, na qual se observa que, embora esta seja a única opção individual viável para redução do 
nível de pressão sonora elevado, são constatadas a presença de dores na região pré-auricular e na articulação temporomandibular (ATM) em alguns colaboradores das empresas, o que pode promover a retirada constante dos equipamentos durante a jornada de trabalho, aumentando assim a incidência de perda auditiva ${ }^{5}$.

A Disfunção temporomandibular (DTM) é definida como uma disfunção na ATM, compreendendo desordens músculo-esqueléticas nas estruturas associadas ou ambos. Com relação à etiologia, apresenta-se multifatorial, com aspectos referentes à oclusão dentária, psicológicos, traumas, hábitos parafuncionais e condições sistêmicas ${ }^{6}$.

Sobre a classificação propõe-se que as desordens intra-articulares acometem a parte interna da articulação e caracterizam-se por lesões e deslocamentos no disco articular. Já as desordens musculares compreendem as alterações nos músculos mastigatórios, neste grupo são enquadrados o espasmo muscular, a contração muscular de proteção, mialgia, fibromialgia e a síndrome da dor miofacial esta é considerada como um conjunto de desordens clínicas músculo-esqueléticas que envolve a musculatura mastigatória, articulação temporomandibular e estruturas associadas. Pode ter sua origem por doenças infecciosas, vasculares, neoplásicas e estar associada a danos teciduais na musculatura mastigatória, que compõem um dos subgrupos da DTM ${ }^{6,7}$.

Devido ao alto índice de funcionários que trabaIham expostos ao ruído intenso, fazendo uso de EPl's do tipo concha, apresentarem queixas de dores na região pré-auricular e na ATM durante e após o uso destes ${ }^{8}$ faz-se necessária a investigação acerca da presença de DTM's. Desta forma, o presente estudo objetivou identificar a presença da DTM em usuários deste equipamento.

\section{MÉTODO}

Tratou-se de uma pesquisa de série de casos descritiva, com caráter observacional, desenvolvida em uma indústria de tintas, com nível médio de ruído de 90 dBNA.

A partir da população de 281 trabalhadores que participaram de exames relativos à saúde ocupacional, identificou-se que 80 colaboradores queixaram-se de desconforto na região da ATM e pré-auricular por uso de DPAI do tipo concha. Foram convidados a participar do estudo todos os trabalhadores, de ambos os sexos, com queixa de desconforto pelo uso do equipamento de proteção auditiva individual (DPAI) (abafador do tipo concha) de qualquer origem.

Para a confirmação da queixa dolorosa e investigação da presença de sinais otológicos realizou-se uma triagem fonoaudiológica com os participantes (Figura 1). Posteriormente os participantes foram submetidos à avaliação otorrinolaringológica com objetivo de excluir participantes que apresentassem problemas otológicos com sintomatologia dolorosa o que, por sua vez, poderia confundir-se com o diagnóstico de DTM comprometendo os resultados (Figura 2). Em apenas um participante encontrouse otite média unilateral.

Desta forma, a amostra final constou de 46 colaboradores, sendo 43 homens e 3 mulheres com idade entre 20 e 50.

A média da idade em anos da população foi de 36,24 com desvio padrão de $\pm 9,57$, o gênero predominante foi o masculino $(94,44 \%)$, e utilizavam DPAl's do tipo concha (abafadores) por 8 horas por dia.

Para verificação de DTM, o Research Diagnostic Criteria For Temporomandibular Disorders (RDC/ TMD) eixo I ${ }^{9}$ (Figura 3) foi aplicado por um fonoaudiólogo pesquisador experiente. O protocolo que consta de um conjunto de critérios de diagnóstico relativos a limitações funcionais e alterações estruturais, para fins de pesquisa. Este envolve a realização de procedimentos e exame clínico da articulação temporomandibular.

O estudo foi desenvolvido obedecendo aos critérios éticos determinados pela Resolução 196/96 que emanam diretrizes para a pesquisa envolvendo seres humanos, na qual o projeto recebeu aprovação pelo Comitê de Ética do Hospital Agamenon Magalhães sob o protocolo $n^{\circ} 57 / 2009$. Todos os voluntários assinaram um termo de consentimento livre e esclarecido expondo assim sua permissão em participar do estudo.

Os dados foram tabulados com auxílio do software SPSS na versão 13.0. Para a análise estatística adotou-se a descrição da freqüência percentual e relativa dos dados. 
FICHA DE TRIAGEM

\section{Dados de Identificação}

Nome:

Empresa:

Idade: Sexo:

Data de Nascimento:

Tempo de Serviço:

RG:

Função: Setor:

\section{Histórico Ocupacional}

1- Trabalhou em ambiente ruidoso anteriormente?

( ) $\operatorname{sim}($ ) não

2- Se sim, há quanto tempo?

3- Fazia uso de EPI?

( ) $\operatorname{sim}($ ) não

4- Qual tipo e modelo

5- Atualmente qual tipo e modelo de EPAI que utiliza

6- Sente dor ou desconforto na região pré-auricular ao usar o EPAI?

( ) $\operatorname{sim}($ ) não

7-Após o uso continua sentindo dores?

( ) $\operatorname{sim}($ ) não

8- Com quanto tempo passa?

( ) um dia

( ) dois dias

( ) três dias

( ) mais de três dias

9- Você tem dor no ouvido?

( ) nenhum 1

( ) direito 2

( ) esquerdo 3

( ) ambos 4

10- Já escorreu alguma coisa do seu ouvido?

( ) nenhum 0

( ) direito 1

( ) esquerdo 2

( ) ambos 3 
11- Sente zumbido no ouvido?

( ) nenhum 0

( ) direito 1

( ) esquerdo 2

( ) ambos 3

12- Que tipo de zumbido?

( ) chiados

( ) apitos

( ) cachoeira

( ) sensação de ouvido cheio

( ) som de cigarra

13- Já realizou alguma cirurgia no ouvido ou próximo a ele?

( ) nenhum 0

( ) direito 1

( ) esquerdo 2

( ) ambos 3

14- Que tipo de cirurgia?

Figura 1 - Ficha de Triagem 


\section{AVALIAÇÃO OTORRINOLARINGOLÓGICA}

\section{Anamnese}

Já teve infecções repetidas das orelhas?

Qual tipo de atividade / função que você exerce?

Tem história familiar de perda auditiva?

Tem algum tipo de doença sistêmica associada (diabetes, hipertireoidismo)?

Usa algum tipo de medicamento?

Possui alguma alteração do sistema auditivo (audição, equilíbrio)?

\section{Exame Físico}

Presença de alterações no pavilhão auditivo externo

( ) $\operatorname{sim}($ ) não

Alteração anatômica ( )

Descreve:

Deformidades ( )

Descreve:

Lesões neoplásicas ou inflamatórias

( ) $\operatorname{sim}($ ) não

Descreve:

Meatoscopia

Orelha direita ( ) normal ( ) alterado

Orelha Esquerda ( ) normal ( ) alterado

Perfuração da membrana timpânica

Orelha direita ( ) sim ( ) não

Orelha Esquerda ( ) sim ( ) não

Audiometria Tonal e Vocal (laudo obtido por meio dos periódicos realizados pela empresa).

Resultado:

Figura 2 - Avaliação Otorrinolaringológica 


\section{RDC/TMD EIXO I - Formulário de Exame}

Nome:
Data:

Pront:

1. Você tem dor no lado direito da sua face, lado esquerdo ou ambos os lados?

nenhum 0

direito 1

esquerdo 2

ambos 3

2. Você poderia apontar as áreas aonde você sente dor?

\begin{tabular}{|l|l|l|l|}
\hline Direito & Esquerdo \\
\hline Nenhuma & 0 & Nenhuma & 0 \\
\hline Articulação & 1 & Articulação & 1 \\
\hline Músculos* & 2 & Músculos* & 2 \\
\hline Ambos & 3 & Ambos & 3 \\
\hline
\end{tabular}

* Especificar o local:

Examinador apalpa a área apontada pelo paciente, caso não esteja claro se é dor muscular ou articular.

\section{Padrão de Abertura}

Reto

Desvio lateral direito (não corrigido) 1

Desvio lateral direito corrigido ("S") 2

Desvio lateral esquerdo (não corrigido) 3

Desvio lateral esquerdo corrigido ("S") 4

Outro

Tipo (especifique)

4. Extensão de movimento vertical: Marcar os incisivos maxilares utilizados 11 ou 21
a. Abertura sem auxílio sem dor $\mathrm{mm}$
b. Abertura máxima sem auxílio $\mathrm{mm}$
c. Abertura máxima com auxílio $\mathrm{mm}$
d. Transpasse incisal vertical $\mathrm{mm}$

Tabela abaixo: Para os itens "b" $e$ "c" somente

\begin{tabular}{|l|c|c|c|c|c|c|c|c|}
\hline & \multicolumn{4}{|c|}{ DOR MUSCULAR } & \multicolumn{4}{c|}{ DOR ARTICULAR } \\
\hline & nenhuma & direito & esquerdo & ambos & nenhuma & direito & esquerdo & ambos \\
\hline b. & 0 & 1 & 2 & 3 & 0 & 1 & 2 & 3 \\
\hline c. & 0 & 1 & 2 & 3 & 0 & 1 & 2 & 3 \\
\hline
\end{tabular}

5. Ruídos articulares (palpação)

a. Abertura

Nenhum

Estalido

Crepitação grosseira

Crepitação fina

$\begin{array}{cc}\text { DIREITO } & \text { ESQUERDO } \\ 0 & 0 \\ 1 & 1 \\ 2 & 2 \\ 3 & 3\end{array}$

Medida do estalido na abertura $\mathrm{mm}$ $\mathrm{mm}$ 
b. Fechamento

Nenhum

DIREITO ESQUERDO

Estalido

Crepitação grosseira

Crepitação fina

$\begin{array}{ll}0 & 0 \\ 1 & 1 \\ 2 & 2 \\ 3 & 3\end{array}$

Medida do estalido no fechamento $\mathrm{mm}$ $\mathrm{mm}$

c. Estalido recíproco eliminado durante abertura protrusiva DIREITO ESQUERDO

Sim $0 \quad 0$

Não 11

$\begin{array}{lll}\text { NA } & 8 & 8\end{array}$

\section{Excursões}

a. Excursão lateral direita $\mathrm{mm}$

b. Excursão lateral esquerda $\mathrm{mm}$

c. Protrusão $\mathrm{mm}$

Tabela abaixo: Para os itens "a", "b" e "c"

\begin{tabular}{|l|c|c|c|c|c|c|c|c|}
\hline & \multicolumn{4}{|c|}{ DOR MUSCULAR } & \multicolumn{4}{c|}{ DOR ARTICULAR } \\
\hline & nenhuma & direito & esquerdo & ambos & nenhuma & direito & esquerdo & ambos \\
\hline a. & 0 & 1 & 2 & 3 & 0 & 1 & 2 & 3 \\
\hline b. & 0 & 1 & 2 & 3 & 0 & 1 & 2 & 3 \\
\hline c. & 0 & 1 & 2 & 3 & 0 & 1 & 2 & 3 \\
\hline
\end{tabular}

d. Desvio de linha média $\mathrm{mm}$

\begin{tabular}{|c|c|c|}
\hline direito & esquerdo & NA \\
\hline 1 & 2 & 8 \\
\hline
\end{tabular}

7. Ruídos articulares nas excursões

Ruídos direito

\begin{tabular}{|l|c|c|c|c|}
\hline & nenhum & estalido & $\begin{array}{c}\text { Crepitação } \\
\text { grosseira }\end{array}$ & $\begin{array}{c}\text { Crepitação } \\
\text { leve }\end{array}$ \\
\hline Excursão Direita & 0 & 1 & 2 & 3 \\
\hline Excursão Esquerda & 0 & 1 & 2 & 3 \\
\hline Protrusão & 0 & 1 & 2 & 3 \\
\hline
\end{tabular}

Ruídos esquerdo

\begin{tabular}{|l|c|c|c|c|}
\hline & nenhum & estalido & $\begin{array}{c}\text { Crepitação } \\
\text { grosseira }\end{array}$ & $\begin{array}{c}\text { Crepitação } \\
\text { leve }\end{array}$ \\
\hline Excursão Direita & 0 & 1 & 2 & 3 \\
\hline Excursão Esquerda & 0 & 1 & 2 & 3 \\
\hline Protrusão & 0 & 1 & 2 & 3 \\
\hline
\end{tabular}


INSTRUÇÕES, ÍTENS 8-10

O examinador irá palpar (tocando) diferentes áreas da sua face, cabeça e pescoço. Nós gostaríamos que você indicasse se você não sente dor ou apenas sente pressão (0), ou dor (1). Circule o número que corresponde a quantidade de dor que você sente. Nós gostaríamos que você fizesse uma classificação separada para as palpações direita e esquerda.

$0=$ Sem dor $/$ somente pressão

$1=$ Dor

8. Dor muscular extra-oral com palpação

a. Temporal (posterior)

DIREITO ESQUERDO "parte de trás da têmpora"

b. Temporal (médio)

"meio da têmpora"

c. Temporal (anterior) "parte anterior da têmpora"

d. Masseter (superior) "bochecha/abaixo do zigoma"

e. Masseter (médio) "bochecha/lado da face"

f. Masseter (inferior) "bochecha/linha da mandíbula"

g. Região mandibular posterior (estilo-hióide/região posterior do digástrico) "mandíbula/região da garganta"

h. Região submandibular (pterigoide medial/supra-hióide/região anterior do digástrico) "abaixo do queixo"

9. Dor articular com palpação
a. Polo lateral "por fora"
b. Ligamento posterior "dentro do ouvido"

\begin{tabular}{|c|c|}
\hline DIREIT & ESQUERDO \\
\hline $\begin{array}{ll}0 & 1\end{array}$ & $\begin{array}{ll}0 & 1\end{array}$ \\
\hline 0 & 0 \\
\hline
\end{tabular}

10. Dor muscular intra-oral com palpação
a. Área do pterigoide lateral "atrás dos molares superiores"
b. Tendão do temporal "tendão"

$\begin{array}{cccc}\text { DIREITO } & \text { ESQUERDO } \\ 0 & 1 & 0 & 1 \\ 0 & 1 & 0 & 1\end{array}$

Figura 3 - Ficha de avaliação Diagnóstica de DTM 


\section{RESULTADOS}

A caracterização e distribuição das queixas apresentadas pelos trabalhados a respeito do uso do DPAI está disposta na Tabela 1.

Tabela 1 - Distribuição dos resultados quanto descrição das queixas de desconforto por uso de Equipamentos de proteção individual auditivo (DPAI) do tipo concha

\begin{tabular}{lcc}
\hline QUEIXA & N & $\%$ \\
\hline $\begin{array}{l}\text { Dor na região que } \\
\text { envolve a orelha e }\end{array}$ & 40 & $87 \%$ \\
$\begin{array}{l}\text { Articulação } \\
\text { temporomandibular }\end{array}$ & 1 & $2 \%$ \\
$\begin{array}{l}\text { Plenitude Auricular } \\
\begin{array}{l}\text { Prurido } \\
\text { Dor na região que } \\
\text { envolve a orelha }\end{array} \\
\begin{array}{l}\text { Articulação } \\
\text { tempormandibular e } \\
\text { prurido }\end{array}\end{array}$ & 1 & $2 \%$ \\
\hline TOTAL & 4 & $9 \%$ \\
\hline & $\mathbf{4 6}$ & $\mathbf{1 0 0}$ \\
\hline
\end{tabular}

Do total da amostra 87\% (36 participantes) já apresentavam algum sintoma doloroso após o uso o DPAI, e obtiveram diagnóstico positivo de DTM, segundo o RDC/TMD Eixo I, conforme descrito a seguir (Tabela 2)

Tabela 2 - Distribuição dos resultados quanto ao diagnóstico de Disfunção Temporomandibular segundo Research Diagnostic Criteria For Temporomandibular Disorders (RDC/TMD) eixo I

\begin{tabular}{lcc}
\hline DIAGNÓSTICO & N & $\%$ \\
\hline $\begin{array}{l}\text { Dor Miofacial } \\
\begin{array}{l}\text { Dor Miofacial com } \\
\text { limitação de abertura oral }\end{array}\end{array}$ & 30 & 83,0 \\
$\begin{array}{l}\text { Dor Miofacial associada a } \\
\text { deslocamento de disco } \\
\text { com redução }\end{array}$ & 2 & 11,0 \\
\hline TOTAL & $\mathbf{3 6}$ & $\mathbf{1 0 0}$ \\
\hline
\end{tabular}

A dor miofacial apresentou-se mais freqüente na população (83\%), já a dor miofacial com limitação foi observada em apenas $11 \%$. O diagnóstico menos evidenciado no estudo foi o deslocamento de disco que corresponde aos desarranjos intraarticulares, que foi percebido em apenas $6 \%$ do total da amostra.
Com relação ao tempo de profissão dos participantes com diagnóstico positivo de DTM observase que a maioria tem tempo de serviço de um a cinco anos $(36,1 \%)$ seguidos de participantes com 11 a 15 anos de profissão fazendo uso de DPAI tipo concha (Tabela 3).

Tabela 3 - Distribuição dos resultados quanto ao tempo de serviço

\begin{tabular}{lcc}
\hline TEMPO DE SERVIÇO & $\mathbf{N}$ & $\%$ \\
\hline$<1$ ano & 6 & 16,7 \\
1 a 5 anos & 13 & 36,1 \\
6 a 10 anos & 6 & 16,7 \\
11 a 15 anos & 8 & 22,2 \\
16 a 20 anos & 1 & 2,8 \\
21 a 25 anos & 1 & 2,8 \\
26 a 30 anos & 1 & 2,8 \\
TOTAL & $\mathbf{3 6}$ & $\mathbf{1 0 0}$ \\
\hline
\end{tabular}

Quanto a relação entre o tempo de uso de DPAI e sinais e sintomas de DTM,observa-se que não houveram associações significativas (Tabela 4).

\section{DISCUSSÃO}

Diversas publicações têm sido relacionadas à investigação dos DPAl's com destaque à segurança, custos e aplicabilidade destes equipamentos ${ }^{10,11}$, porém poucos estudos versam sobre o desconforto destes e de suas implicações devido ao uso prolongado ${ }^{12}$.

Entretanto, é válido ressaltar que apesar disto, obteve-se um índice considerável de DTM nesta população (87\%). Estes resultados mostram-se com alta prevalência o que nos remete a possibilidade dos DPAl's do tipo concha interferirem consideravelmente no quadro doloroso.

Dos colaboradores estudados, 36,1\% tinha tempo de serviço entre um a cinco anos, o que demonstra possível rotatividade neste segmento. Outro aspecto atribuído a esta variável seria que estudos na área de saúde do trabalhador apontam que quanto maior o tempo de serviço dos colaboradores, maiores são as queixas relativas a desconforto pela quantidade de exposição à pressão e peso destes equipamentos ${ }^{3,8}$.

Destaca-se ainda, nesta pesquisa, a existência de queixas relativas a desconforto pelo uso do DPAl, na qual esta variável mostrou-se presente em todos os casos investigados. Dentre as características mais freqüentes, observou-se a dor na região pré-auricular e na articulação temporomandibular, 
Tabela 4 - Tempo de trabalho com uso de DPAI, segundo o tipo de diagnóstico

\begin{tabular}{lccc}
\hline & \multicolumn{2}{c}{ DIAGNÓSTICO DA DOR MIOFACIAL } & \\
\cline { 2 - 3 } ESTATÍSTICA & Dor miofacial & $\begin{array}{c}\text { Dor miofacial com limitação } \\
\text { de abertura bucal } \\
\text { /deslocamentos de disco }\end{array}$ & Valor de $\mathbf{p}$ \\
\cline { 2 - 3 } & $\mathbf{( n = 3 0 )}$ & $\mathbf{( n = 6 )}$ & \\
\hline Média $^{(\mathbf{1})}$ & 5,97 & 13,37 & $\mathrm{p}^{(1)}=0,155$ \\
Mediana $^{(1)}$ & 3,25 & 11,00 & \\
Desvio padrão $^{(1)}$ & 5,09 & 12,30 & \\
\hline
\end{tabular}

(1): Através do teste Mann-Whitney.

o que nos remete a possibilidade destes DPAl's contribuírem para o desencadeamento ou intensificação das dores orofaciais de origem muscular, ou seja, as mialgias mastigatórias que, correspondem à principal causa de dor no segmento orofacial ${ }^{13}$, é um tipo de DTM de alta prevalência, que envolve os músculos temporal, masseter, pterigóideo medial e lateral (músculos mastigatórios) e é classificada como dor somática profunda, caracterizada por estados de fadiga ou dor, agravados pela movimentação passiva ou funcional da mandíbula, podendo produzir uma limitação de abertura bucal, o que se assemelha as características dolorosas descrita pela população.

Os abafadores consistem em protetores auditivos que se configuram por duas calotas posicionadas cada uma em um dos pavilhões auriculares que por sua vez pressionam o pavilhão por meio de uma banda de material plástico que podem ser ajustadas horizontalmente para o controle da pressão, porém o autor destaca que devido a esta pressão que comprime a região diminuindo a irrigação sanguínea podem ocorrem quadros de irritação ou dor na região comprimida ${ }^{14}$.

Outro fator associado ao desconforto seria a relação direta entre a atenuação conferida e o peso do abafador, em que se segue a seguinte premissa: nos ambientes mais ruidosos (como nas fábricas) se preconiza o uso de EPl's mais pesados, que por sua vez atenuam mais o ruído ambiental. Esta característica pode desencadear casos de estresse e agravar o quadro doloroso ou causar sensações de calor e aumentar a transpiração nesta região o que por sua vez pode facilitar quadros de micose (ambiente quente e úmido), justificando a presença de prurido ${ }^{15}$.
Referente à presença de dor na região orofacial observou-se que esta esteve freqüente em 36 dos 46 colaboradores o que reforça a hipótese descrita anteriormente da acentuada presença de DTM nos colaboradores que fazem uso de EPI do tipo concha. Desta forma, foi realizado o diagnóstico onde se comprovou que a maioria da população apresentou quadro de dor miofacial sem limitação de abertura bucal.

Estudos mostram que a pressão na região muscular pode desencadear ou acentuar os quadros de DTM ${ }^{16}$, o que se justifica pelo aumento da pressão dos abafadores na região, estimulando os receptores mecânicos periféricos (mecanismos responsáveis pelo envio da sensação dolorosa). Uma vez aumentada à sensibilidade estes são capazes de transformar em dor os estímulos recebidos o que se configura na formação do quadro álgico ${ }^{16}$.

$\mathrm{Na}$ análise entre o tempo de uso de DPAI e sinais e sintomas de DTM não foram identificadas associações entre as variáveis, o que remete a possibilidade que estudos futuros e mais aprofundados sobre o impacto dos DPAl's nas disfunções temporomandibulares devem ser realizados para possibilitar o uso de estratégias que visem à minimização destes sintomas garantindo aos funcionários uma maior satisfação e conforto.

\section{CONCLUSÕES}

A partir da realização deste estudo pode-se observar e classificar a presença de DTM muscular caracterizada por dor miofacial na maioria dos usuários de DPAl tipo concha. 


\section{ABSTRACT}

Purpose: to describe and classify the presence of temporomandibular joint disorders in workers using individual hearing protection equipment. Method: this is a transversal descriptive study, with observational character, developed in a paint industry. The population was composed of 46 employees of both genders (94.44\% of which were male) aged between 20 and 50 years (average 36.24-year old), exposed to hazardous noise (90 dBNA) that had been using personal protective equipment (ear muff). In collecting the data, the following procedures were made: speech pathology screening of the oral functions, otorhinolaryngological evaluation and diagnosis of temporomandibular joint dysfunction by using a clinical examination. Data were analyzed using descriptive statistics. Results: the main complaint was pain around the ear and temporomandibular joint. It was observed that $87 \%$ showed a positive diagnosis of TMD while $83 \%$ had myofacial pain, $11 \%$ myofacial pain with oral opening limitation and $6 \%$ showed myofacial pain diagnosis associated with disc displacement with reduction. Conclusions: the majority of the screened workers showed dysfunctions of muscular order. The possibility that the use of this equipment may influence the occurrence or intensification of the mentioned dysfunction must be emphasized.

KEYWORDS: Temporomandibular Joint Disorders; Facial Pain; Occupational Health; Hearing Protective Devices

\section{REFERÊNCIAS}

1. Dias A, Cordeiro R, Corrente JE, Gonçalves CGO. Associação entre perda auditiva Induzida pelo ruído e zumbidos, Rev. Cad. Saúde Publica. 2006;22(1):63-8.

2. Gonçalves CGO, Couto CM, Carraro, JM, Leonelli BS. Avaliação da colocação de protetores auriculares em grupos com e sem treinamento. Rev. CEFAC. 2009; 2(11): 345-52

3. Arezes PM, Miguel ASR. Influência do conforto na eficiência da protecção individual auditiva. Rev Tecni Acústica.1999. [cited 2010 Dez 10]. Available from: http://www.sea-acustica.es/ publicaciones/4366vt008.pdf

4. Otoni AM, Boger E, Barbosa-Branco A, Shimizu HE, Maftum MA. Ruído ocupacional como fator de risco para perda auditiva Rev Cogitare Enferm. 2008; 13(3):367-73.

5. Boger ME, Barbosa-Branco A, Ottoni ÁC. A influência do espectro de ruído na prevalência de perda auditiva induzida por ruído em trabalhadores, Rev Braz. j. otorhinolaryngol.2009; 75(3):328-34. 6. Martins RJ, Garcia AR, Garbin CAS, Sundefeld MLMM. Associação entre classe econômica e estresse na ocorrência da disfunção temporomandibular. Rev. bras. epidemiol. [periódico na internet]. 2007 [acesso em 2010 ago 24]; 10(2):21522. Disponível em: http://www.scielo.br/pdf/rbepid/ v10n2/08.pdf.

7. Gomes MB, Guimarães FCS, Guimarães M RA, Claro Neves C. Limiar de dor à pressão em pacientes com cefaléia tensional e disfunção
Temporomandibular. Rev Cienc Odontol Bras. 2006; 9 (4): 84-91.

8. Abelenda C. Avaliação do conforto dos protectores individuais auditivos.[dissertação]. Portugal, Universidade do Minho. - Faculdade de Engenharia Humana; 2006.

9. Dworkin SF, Le Resche L. Research diagnostic criteria for temporomandibular disorders: review, criteria, examinations and specifications, critique. Rev. J Craniomandib Disord. 1992;6 (4):301-55.

10. Ribeiro AMD,Camara VM.Perda auditiva neurossensorial por exposição continuada a níveis elevados de pressão sonora em trabalhadores de manutenção de aeronaves de asas rotativas. Rev Cad. Saúde Pública. 2006; 22(6):1217-24.

11. Azevedo AN, Bernardo LD, Shing SCAC,Santos JN. Perfil auditivo de trabalhadores de um entreposto de carnes. Rev. CEFAC. 2010; 12(2)223-34.

12. Santoni $\mathrm{CB}$, Fiorini $\mathrm{ACl}$. Músicos de pop-rock: avaliação da satisfação com protetores auditivos, Rev Braz. j. otorhinolaryngol.2010;76(4): 454-61.

13. Pimentel PHWG, Coelho Júnior LGTM, Caldas Júnior AF, Kosminsky M, Aroucha JMCNL. Perfil demográfico dos pacientes atendidos no Centro de Controle da Dor Orofacial da Faculdade de Odontologia de Pernambuco Rev. cir. traumatol. buco-maxilo-fac. 2008;8(2):69-76.

14. Rodrigues MAG, Dezan AA, Marchiori LLM. Eficácia da escolha do protetor auditivo pequeno, médio e grande em programa de conservação auditiva. Rev. CEFAC. 2006, 4 (8) 543-7 
15. Barros MVG, Nahas MV. Comportamentos de risco, auto-avaliação do nível de saúde e percepção de estresse entre trabalhadores da indústria. Rev. Saúde Pública. 2001;35(6):554-63.
16. Silva ROF, Conti PRSS, Araújo CRP. Quantidade de pressão e padrão de dor referida em pacientes portadores de Dor Miofascial. Rev. Robrac. 2007;16(42): 1-8.

http://dx.doi.org/10.1590/S1516-18462011005000056

RECEBIDO EM: 06/07/2010

ACEITO EM: 30/12/2010

Endereço para correspondência:

Hilda Santos de Souza Mendes Aquino

IDE Instituto de Desenvolvimento Educacional

Rua dos navegantes 992

Boa Viagem, Recife

CEP: 51021010

E-mail: ferri-representacoes@gmail.com silviabenevides@ufba.br tatianapss2@gmail.com 